\title{
SIMULTANEOUS ESTIMATION OF FUNCTIONAL RELATIONSHIP AMONG NON-DIMENSIONAL VARIABLE GROUPS AND ITS COEFFICIENTS IN ANALYZING EXPERIMENTAL DATA
}

\author{
HaJIME UNNO AND TAKASHI AKEHATA \\ Department of Environmental Chemistry and Engineering, \\ Tokyo Institute of Technology, Yokohama 227
}

\begin{abstract}
A method of correlating data in terms of non-dimensional variable groups is discussed. The method is based on Group Method of Data Handling (GMDH). An algorithm for the method is developed and an example of the algorithm's application is discussed. The algorithm developed is convenient for correlating experimental data of complicated systems. It should be stressed that the application of the method necessitates many kind of heuristics through which the method shows the versatility in the application.
\end{abstract}

\section{Introduction}

Dimensional analysis has been used for correlating experimental data of complicated systems. However, it is recognized that no definite functional relationship among variables concerned is revealed by the method. Moreover, in determining the concrete functional relationship a quantity of data set over a sufficiently wide range of the condition should be available.

In most dimensional analysis the relationship among the non-dimensional variable groups is elucidated step by step. The procedure is that, first, the relationship between two non-dimensional variable groups is examined while holding all the other groups constant; second, the relationship between one of the two groups already examined and one of the remaining groups is examined, with the values of all the other groups being held constant; and so on. Therefore, cases sometimes occur in which arrayed data in the form of non-dimensional variable groups are not sufficient though the amount of data in the form of dimensional variables are abundant. In the light of these facts a method is proposed for simultaneous estimation of the functional relationship among nondimensional variable groups and the coefficients included in it.

1. Concept of GMDH and Applicability of Its Technique to Data Processing in Terms of Non-dimensional Variable Groups

As described above it is desirable to develop a

Received July 5, 1979. Correspondence concerning this article should be addressed to $\mathrm{H}$. Unno. method of correlation among non-dimensional variable groups where the data are not sufficient in number for the usual correlation method. A method meeting this requirement is suggested from the concept of group method of data handling (GMDH) developed by Ivakhnenko $o^{3,4}$. The principle of GMDH is based on the art of "improvement of breed". A breed is improved by human experience over a long time, and its effectiveness has been demonstrated for the improvement of both plants and animals. Actually, improvement of a breed is performed by first selecting seeds of superior character. Many random pairs of seeds are "crossbred" in the second step. Among the new seeds that result, those of superior character are selected for the next "crossbreeding" in the third step, and so on.

GMDH is one of the techniques developed for the analysis or identification of complicated systems, the behavior of which is too hopelessly entangled to be analysed by systematic mathematical techniques such as a set of differential equations. In other words, GMDH is a general method of handling data of a system having the following characteristics.

1) The system has many variables.

2) The interrelations among the variables are not known and the system may include nonlinear relationship.

3) The causality among the variables is not clear.

These are just the characteristics contained within the dimensional analysis. This fact suggests the applicability of GMDH to the data processing of complex systems.

Although the theoretical background of GMDH has 


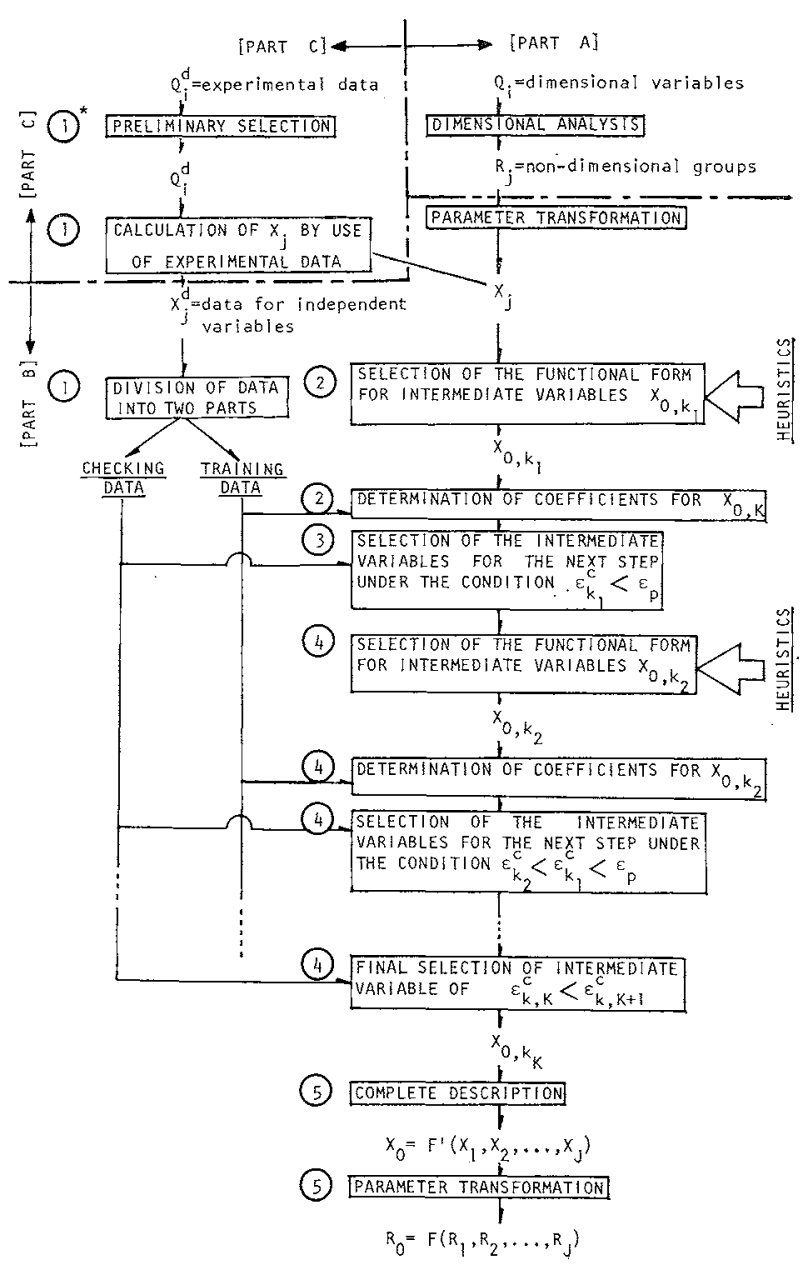

Circled number shows the procedure step in the text.

Fig. 1 General algorithm for dimensional analysis by use of GMDH

not thoroughly been made clear yet, the effectiveness of the method has been shown in the data processing of several complex systems, such as those for environmental control and/or estimation, management and industrial processes ${ }^{2,5}$.

2. Algorithm for Simultaneous Estimation of Functional Relationship and Its Optimum Parameters at the Same Time

Figure 1 shows the general configuration of the algorithm for the method of determining both the functional relationship and its parameters at the same time. Part A of Fig. 1 shows a procedure of finding out the non-dimensional variable groups associated with the phenomena concerned. This is the same procedure that is followed in conventional dimensional analysis. Part B of Fig. 1 shows the procedure of searching the functional relationship among the nondimensional variable groups found in Part $\mathrm{A}$. The procedure of Part B needs some "heuristics". "Heuristics" refers to the way of selecting an elementary function which constitutes intermediate variables.
The non-dimensional variable groups found in Part A will play a role of seeds for the first "crossbreeding" in the procedure of "improvement of breed". The intermediate variables to be selected and the "heuristics" correspond to the seeds resulting from the "crossbreeding" and the way in which the "crossbreeding" will be conducted, respectively.

The selection of elementary function is the most important procedure of the technique. This selection requires some physical intuition involved in the phenomena and/or some other information such as whether the functional relationship for the approximation is given beforehand or not.

Part C of Fig. 1 shows the scheme of data processing.

General procedures of data analysis shown in Fig. 1 are as follows.

[Procedure 1] Calculation of nON-Dimensional VARIABLE GROUPS BY USE OF EXPERIMENTAL DATA

It is sometimes useful to make a preliminary selection of data by physical intuition, for example, by discarding the data obtained under poor conditions. Let $R_{j}^{d}, J+1$ and $n_{j}$ be numerical data of $j$-th nondimensional variable group calculated by experimental data, number of non-dimensional variable groups and number of the data, respectively. The $n_{j}(j=1,2, \ldots$, $J)$ data of $R_{j}^{d}(j=1,2, \ldots, J)$ serve as original data. The $n_{j}$ original data of non-dimensional variable groups $R_{j}^{d}$ are divided into two groups. One of the two groups serves as "training data" and the other is used as "checking data". The method of division is not unique, but the principle of equal statistical character for both groups of data is recommended. It should be stressed that the data division is one of the features of GMDH.

If a functional relationship is postulated beforehand the original data must be transformed. For example, consider the case in which the functional relationship is approximated by Eq. (1).

$$
R_{0}=A R_{1}^{a_{1}} R_{2}^{\alpha_{2}} \ldots R_{J}^{a_{J}}
$$

where $R_{j}(j=1,2, \ldots, J)$ are non-dimensional variable groups and the optimal values of $A$ and $a_{j}(j=1,2, \ldots$, $J)$ are to be estimated by the experimental data, $R_{j}^{d}$. In the dimensional analysis of almost all cases the functional relationship of Eq. (1) is postulated. Although there are many successful cases of correlating data in the form of Eq. (1), the selection of the functional form of Eq. (1) is not made from the view point that it is optimal. Moreover, even if the functional relationship of Eq. (1) is selected, determining the optimal values of parameters $A$ and $a_{j}$ is not easy work.

Under the condition of the postulated functional form of Eq. (1) the following transformation is necessary.

$$
X_{j}=\ln R_{j}
$$


This is because the transformed equation expresses linear combinations of $X_{j}$ as

$$
X_{0}=\ln A+a_{1} X_{1}+a_{2} X_{2}+\ldots+a_{J} X_{J}
$$

The transformed variables $X_{j}(j=1,2, \ldots, J)$ are processed under the following procedure.

[Procedure 2] SELECTION OF FUNCTIONAL FORM FROM THE INTERMEDIATE VARIABLES

The functional form of the intermediate variables $X_{0, k}(k=1,2, \ldots)$ are selected based on the "heuristics". The intermediate variables $X_{0, k}$ are functions of all possible combinations of the two variables $X_{i}$ and $X_{j}$. One of the useful functions including nonlinear relation is the following simplest combination of the two variables $X_{i}$ and $X_{j}$. This procedure corresponds to "crossbreeding".

$$
X_{0, k}=b_{0(i j)}^{k}+b_{1(i j)}^{k} X_{i}+b_{2(i j)}^{k} X_{j}+b_{3(i j)}^{k} X_{i} X_{j}
$$

In the case of the postulated final form of Eq. (1), the intermediate variables of Eq. (5) are adopted under the condition of the data transformation through Eq. (2).

$$
X_{0, k}=b_{0(i j)}^{k}+b_{1(i j)}^{k} X_{i}+b_{2(i j)}^{k} X_{j}
$$

This is due to the fact that recombination of the intermediate variables $X_{0, k}$ defined by Eq. (5) also produces a linear combination of intermediate variables $X_{j}$ and the final expression is the linear form of Eq. (3).

The coefficients $b_{l(i j)}^{k}$ are determined by the "training data" so as to minimize the relative mean square error of Eq. (6).

$$
\varepsilon_{k}^{t}=\sum_{m=1}^{M^{t}}\left(1-\frac{R_{0, k}}{R_{0, m}^{d, t}}\right)^{2}
$$

where $R_{0, k}, R_{0, m}^{d, t}$ and $M^{t}$ are the left-hand side of correlation equation calculated through Eq. (4) or (5), the training data of $R_{0}$ and the number of training data set, respectively. This is the procedure equivalent to the "selection of seeds of superior character". [Procedure 3] Selection of INTERMediate Variable

The checking data are used to evaluate the relative mean square error of Eq. (7) for each variable combination the coefficient of which is determined in PROCEDURE 2.

$$
\varepsilon_{k}^{c}=\sum_{n=1}^{M^{c}}\left(1-\frac{R_{0, k}}{R_{0, n}^{d, c}}\right)^{2}
$$

where $R_{0, m}^{d, e}$ and $M^{c}$ are the checking data of $R_{0, k}$ and the number of checking data set, respectively. The selection of intermediate variables are made by the criterion that the calculated relative mean square error is less than a predetermined value, or the criterion that a predetermined number of intermediate variables have smaller mean square errors.

[Procedure 4] Selection OF NeXt intermediate VARIABLES

The variables selected as $X_{0, i}$ and $X_{0, j}$ are regarded as $X_{i}$ and $X_{j}$ in the Procedure 2 , respectively. Then
Procedures 2 and 3 are repeated. In this procedure a new description for $X_{0, k}$ may be possible if it is necessary. This is another "heuristic". These procedures are repeated until the time when the minimum of the checked relative mean square error of Eq. (7) decreases no further. The value of $X_{0, k}$ of minimum error is the final selection.

\section{[Procedure 5] COMPlete description}

After the final selection of the intermediate variables the complete description of variables is obtained by inserting the intermediate variables selected into the former ones selected in the iteration successively. The complete description is not unique if the final functional form is not preliminarily determined, because the intermediate variable can take any functional form.

\section{Algorithm for a Postulated Functional Form}

Nowadays any complex description can be treated by a high-speed computer. However, too complex a description is not suitable for understanding the physical interrelation of non-dimensional variable groups or dimensional variables. This fact suggests that it is useful to develop an algorithm which can be applied to an assumed functional form.

Consider an algorithm for the following postulated relationship among the non-dimensional variable groups $R_{j}$.

$$
R_{0}=A R_{1}^{a_{1}} R_{2}^{a_{2}} \ldots R_{J}^{a_{J}} \cdot h\left(R_{1}, R_{2}, \ldots, R_{J}\right)
$$

If $h\left(R_{1}, R_{2}, \ldots, R_{J}\right)=1$ this is the case of conventional dimensional analysis. Therefore, the function $h\left(R_{1}\right.$, $R_{2}, \ldots, R_{J}$ ) means the correction with respect to the expression of Eq. (1). One of the functional forms for $h\left(R_{1}, R_{2}, \ldots, R_{J}\right)$ suitable for the approximated correlation Eq. (1) is the following expression.

$$
\begin{aligned}
h\left(R_{1}, R_{2}, \ldots, R_{J}\right)= & 1+\sum_{i} b_{i} R_{i}+\sum_{i j} b_{i j} R_{i} R_{j} \\
& +\sum_{i j k} b_{i j \mid b} R_{i} R_{j} R_{l k}+\ldots
\end{aligned}
$$

Equations (8) and (9) are lengthy but straightforward expressions. Therefore, they are practicable for the complete description.

Figure 2 shows an algorithm of the method for the final expression of Eqs. (8) and (9). Part A of Fig. 2 is the first approximation procedure, that is, the procedure of searching for the optimal coefficient $A$ and exponents $a_{j}$. Part $B$ of Fig. 2 is the algorithm for self-organization procedure for the correlation function of Eq. (9). The data necessary for the correlation function $h\left(R_{1}, R_{2}, \ldots, R_{J}\right)$ is prepared by transforming the original data through Eq. (10).

$$
h^{d}=\frac{R_{0}}{A R_{1}^{a_{1}} R_{2}^{a_{2}} \ldots R_{J}^{a_{J}}}
$$

The intermediate variables selected here expresses the 


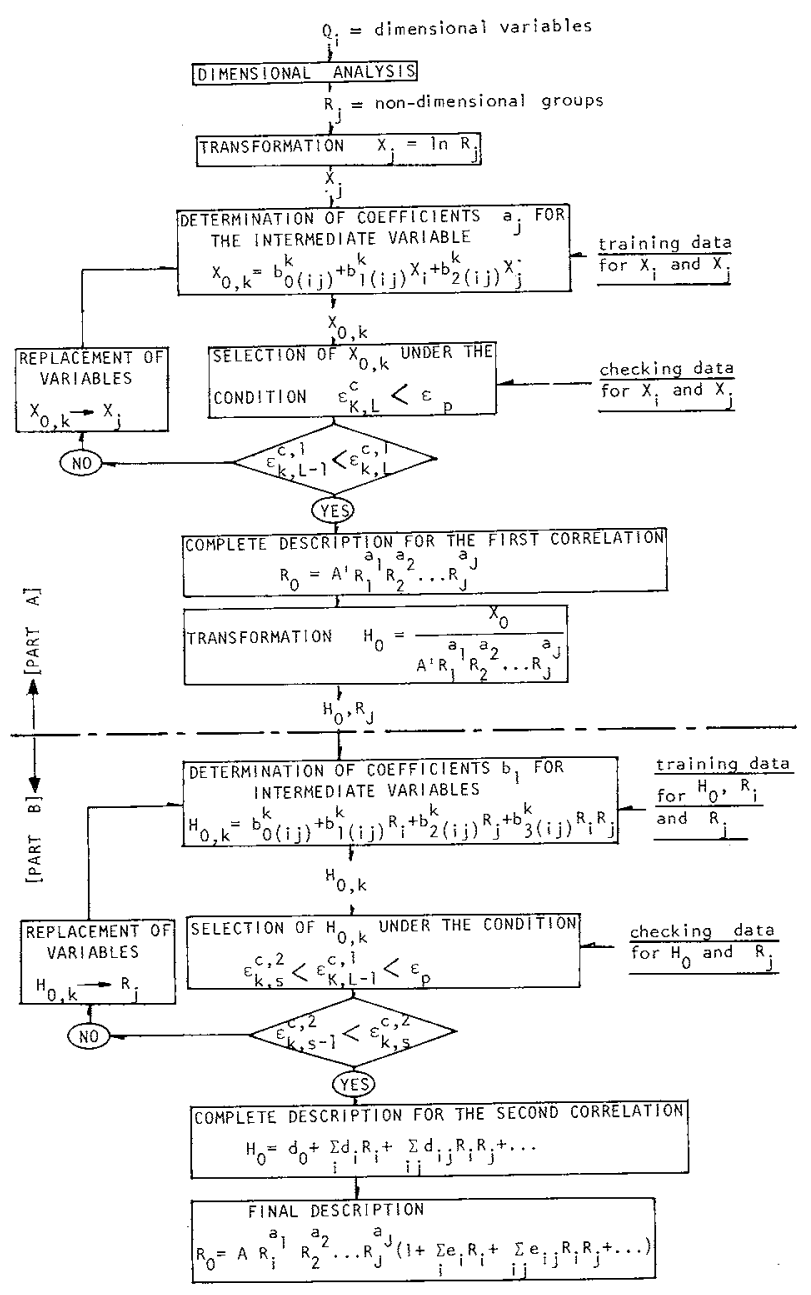

Fig. 2 Algorithm for dimensional analysis under the condition of a postulated functional relationship among non-dimensional variable groups

simplest non-linear form of Eq. (4) because this variable gives the expanded form of any analytical function.

If the relative mean square error $\varepsilon_{k}^{c \cdot 2}(k=1,2, \ldots)$ obtained in Part $B$ of Fig. 2 is greater than $\varepsilon_{1}^{c, 1}$, which is the smallest error obtained in the first approximation, this means that the first approximation gives the best correlation and needs no more calculation.

\section{Example of the Method}

Consider the power requirement data of a surface aerator as analyzed by the proposed method as an example. The experimental data of power consumption of an aeration tank have been discussed elsewhere ${ }^{11}$ in relation to the geometrical and operating conditions. A surface aerator consisting of a cylindrical tank was filled with water and fitted with a turbine-type impeller at the water surface. Physical quantities considered to take part in the power requirement, $P$, are tank diameter, $D$, water depth, $H$, impeller size, i.e., diameter, height and blade width, and impeller rotational speed, $N$, and physical properties of liquid, i.e., vis- cosity, $\mu$, and density, $\rho$. As the experiment was carried out under the geometrical similarity condition of impeller the impeller diameter, $d$, can be selected as a representative size of impeller.

Taking into account the variables described above, dimensional analysis reveals four non-dimensional groups, i.e., power number $Z_{1}\left(=P / \rho N^{3} d^{5}\right)$, Reynolds number $Z_{2}\left(=\rho N d^{2} / \mu\right)$, scale ratios $Z_{3}(=d / D)$ and $Z_{4}(=H / d)$. The optimum functional relationship and its parameters are searched by the algorithm shown in Fig. 2. The results are summarized in Table 1. The minimum error of the first approximation step is obtained by the first selection of original non-dimensional variable groups (No. 1 of comment column in Table 1) and the first approximation is expressed as follows:

$$
Z_{1}=0.845 Z_{2}^{-0.755} Z_{4}^{0.334}
$$

The minimum error obtained by the second approximation (No. 7 of comment column in Table 1) is less than that of the first approximation. Therefore, the final approximation is obtained as follows:

$$
H_{0}=1.09-0.454 Z_{3}+0.353 Z_{3}{ }^{2}
$$

or

$$
\begin{aligned}
P / \rho N^{3} d^{5}= & 0.921\left(\rho N d^{2} / \mu\right)^{-0.755}(H / d)^{0.334} \\
& \times\left\{1.00-0.417(d / D)+0.324(d / D)^{2}\right\}
\end{aligned}
$$

The minimum errors obtained in the first and second correlation steps are nearly equal to each other. Therefore, there arises the problem of optimum selection among the functional relationships for the approximation level. In such a situation the selection should be made from a physical viewpoint or the convenience of usage of the correlation. This can be another "heuristic". In the present example the expression of Eq. (11) will be recommended for simplicity as the precision of the correlation is not greatly increased in the second correlation step.

\section{Concluding Remarks}

An algorithm of data analysis in terms of nondimensional variable groups is developed which is an application of the group method of data handling (GMDH). An application of the method is discussed and it is shown that many kinds of "heuristics" are needed for application.

\section{Nomenclature}

$\begin{array}{llr}A & =\text { coefficient } & {[-]} \\ a & =\text { exponent } & {[-]} \\ b & =\text { coefficient } & {[-]} \\ D & =\text { diameter of aeration tank } & {[\mathrm{m}]} \\ d & =\text { coefficient } & {[-]} \\ & \text { or impeller diameter } & {[\mathrm{m}]} \\ e & =\text { coefficient } & {[-]} \\ H & =\text { liquid depth of aeration tank } & {[\mathrm{m}]} \\ h(. .) & =\text { correction function defined by Eq. (8) } & {[-]}\end{array}$


Table 1 Numerical example of the method

First CORRELATION PROCEDURE (PART A of Fig. 2)

\begin{tabular}{|c|c|c|c|c|c|c|c|}
\hline \multirow{2}{*}{$\begin{array}{c}\text { Approximation } \\
\text { step } \\
n\end{array}$} & \multirow{2}{*}{$\begin{array}{c}\text { Intermediate } \\
\text { variable } \\
Z_{k}^{n}\left(Z_{i}^{n-1}, Z_{j}^{n-1}\right)^{*}\end{array}$} & \multicolumn{4}{|c|}{ Parameters $\left(Z_{k}^{n}=A Z_{2}^{a_{1}^{n}} Z_{3}^{a_{2}^{n}} Z_{4}^{a_{3}^{n}}\right)$} & \multirow{2}{*}{$\begin{array}{l}\text { Error } \\
\varepsilon_{k}^{c, 1}\end{array}$} & \multirow[t]{2}{*}{ Comment } \\
\hline & & $A$ & $a_{1}^{n}$ & $a_{2}^{n}$ & $a_{3}^{n}$ & & \\
\hline \multirow{3}{*}{1} & $Z_{2}^{1}\left(Z_{2}^{0}, Z_{4}^{0}\right)^{* *}$ & 0.845 & -0.755 & 0 & 0.334 & 0.149 & 1 \\
\hline & $Z_{3}^{1}\left(Z_{2}^{0}, Z_{3}^{0}\right)$ & 1.37 & -0.801 & -0.136 & 0 & 0.314 & 2 \\
\hline & $Z_{4}^{1}\left(Z_{3}^{0}, Z_{4}^{0}\right)$ & 0.00173 & 0 & -0.414 & 0.482 & 0.339 & 3 \\
\hline \multirow{3}{*}{2} & $Z_{2}^{2}\left(Z_{2}^{1}, Z_{3}^{1}\right)$ & 0.892 & -0.761 & -0.0152 & 0.269 & 0.161 & 4 \\
\hline & $Z_{3}^{2}\left(Z_{3}^{1}, Z_{4}^{1}\right)$ & 0.439 & -0.708 & -0.226 & 0.123 & 0.213 & 5 \\
\hline & $Z_{4}^{2}\left(Z_{2}^{1}, Z_{4}^{1}\right)$ & 1.32 & -0.724 & 0.0 & 0.320 & 2.28 & 6 \\
\hline
\end{tabular}

SeCond CORRelation PRocedure (PART B of Fig. 2)

$\begin{gathered}\text { Approximation } \\ \text { step } \\ n\end{gathered}$
$Z_{k} \begin{gathered}Z_{k}^{n}\left(Z_{i}^{n-1}, Z_{j}^{n-1}\right)^{*} \\ \text { variable }\end{gathered}$

* $Z_{k}^{n}$ consists of two intermediate variables $Z_{i}^{n-1}$ and $Z_{j}^{n-1}$

** $\quad Z_{i}^{0}(i=1-4)$ are non-dimensional groups $Z_{i}(i=1-4)$

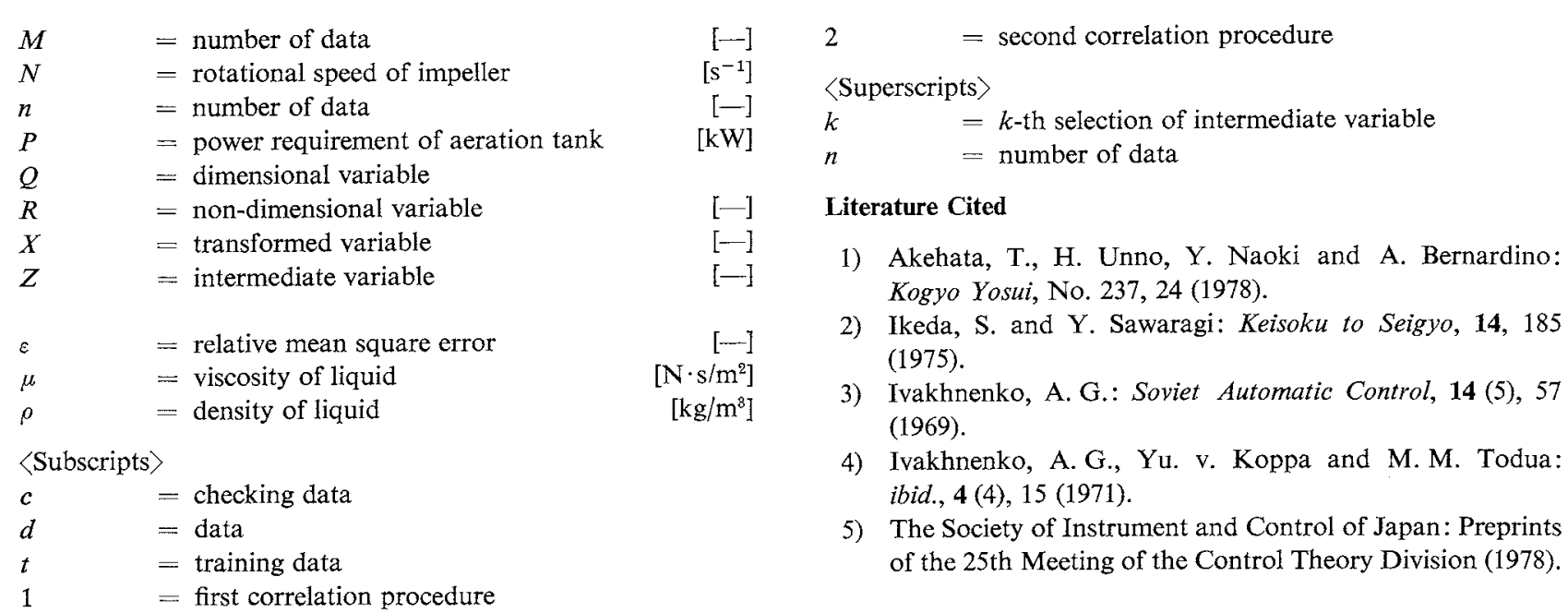

